Copyright (C) 2014 IEEE. Personal use of this material is permitted. Permission from IEEE must be obtained for all other uses, in any current or future media, including reprinting/republishing this material for advertising or promotional purposes, creating new collective works, for resale or redistribution to servers or lists, or reuse of any copyrighted component of this work in other works. 


\title{
Joint Transceiver Optimization for Two-Way MIMO Relay Systems with MSE Constraints
}

\author{
Zhiqiang He, Member, IEEE, Zhouhao Lang, Yue Rong, Senior Member, IEEE, and Sicong Qu
}

\begin{abstract}
Transceiver design for two-way multiple-input multiple-output (MIMO) relay systems has attracted much research interest recently. However, there is little research on the impact of quality-of-service (QoS) constraints on two-way MIMO relay systems, which greatly affects the user experience. In this letter, we propose a transceiver design for two-way MIMO relay systems which minimizes the total network transmission power subjecting to QoS constraints expressed as upper-bounds on the mean-squared error (MSE) of the signal waveform estimation at both destinations. An iterative algorithm is developed to optimize the source, relay, and receive matrices. Simulation results demonstrate the fast convergence of the proposed algorithm.
\end{abstract}

Index Terms-Two-way relay, MIMO relay, QoS, MMSE, linear non-regenerative relay.

\section{INTRODUCTION}

Wireless relaying is a well-known method to extend the cell coverage and increase the network capacity in wireless communication [1]. When multiple antennas are mounted on one or more nodes, we call such relay system a multipleinput multiple-output (MIMO) relay system. As a promising technique for high-speed reliable data transmission, MIMO relay communication has attracted much research interest recently [2], [3].

In a two-way relay communication system, two source nodes exchange their information through an assisting relay node. Compared with other relay strategies such as the digital network coding [4] and the physical network coding [5], the amplify-and-forward (AF) strategy has lower complexity, shorter processing delay, and lower implementation cost. Therefore, the AF strategy is considered in this letter. For two-way AF MIMO relay systems, the optimal relay and source matrices have been developed in [6] to maximize the two-way sum mutual information (MI). Joint source and relay optimization for two-way MIMO relay systems has been studied in [7] to minimize the total mean-squared error (MSE) of the signal waveform estimation. Recently, a unified framework has been developed in [8] to optimize the source and relay matrices for a broad class of objective functions.

The aim of [6]-[8] is to optimize a given objective function, subjecting to the transmission power constraint at each node. However, the quality-of-service (QoS) constraints are not addressed in [6]-[8]. Note that in practical communication systems, QoS criteria are very important, as they greatly affect the user experience. For one-way MIMO relay systems, the QoS-constrained source and relay precoding matrices design

Z. He, Z. Lang, and S. Qu are with the Key Laboratory of Universal Wireless Communication, Ministry of Education, Beijing University of Posts and Telecommunications, Beijing 100876, China (e-mails: hezq; langzhouhao; qscbupt@bupt.edu.cn).

Y. Rong is with the Dept. Electrical and Computer Engineering, Curtin University, Bentley, WA 6102, Australia (e-mail: y.rong@curtin.edu.au).

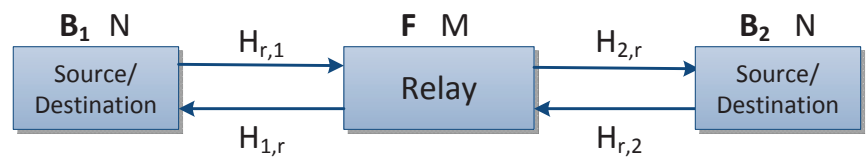

Fig. 1. Block diagram of a two-way MIMO relay communication system.

has been studied in [9]-[11]. However, the approach in [9]-[11] cannot be applied to two-way MIMO relay systems.

In this letter, we propose a transceiver design for AF twoway MIMO relay systems which minimizes the total network transmission power subjecting to QoS constraints, which has not been considered before. Based on the strong link between the MSE and most commonly used MIMO communication system objective functions [8], the QoS criteria are set up as upper-bounds on the MSE of the signal waveform estimation at both destinations. Since the QoS-constrained two-way MIMO relay optimization problem is highly nonconvex with matrix variables, we propose an iterative algorithm to optimize the source, relay, and receive matrices through solving standard convex subproblems, which attains a stationary point (possibly suboptimal) of the problem. Simulation results demonstrate the fast convergence of the proposed algorithm.

\section{SySTEM MODEL}

We consider a two-way MIMO communication system where two nodes exchange information via a relay node as shown in Fig. 1. We assume that $N$ antennas are deployed at each source/destination while $M$ antennas are equipped at the relay node. The communication process is completed in two time slots. At the first time slot, source node $i$ transmits $\mathbf{x}_{i}=\mathbf{B}_{i} \mathbf{s}_{i}, i=1,2$, where $\mathbf{s}_{i}$ is the $N_{b} \times 1\left(N_{b} \leq \min (N, M)\right)$ source signal vector, $\mathbf{B}_{i}$ is the $N \times N_{b}$ source precoding matrix. The received signal vector at the relay node can be written as

$$
\mathbf{y}_{r}=\sum_{i=1}^{2} \mathbf{H}_{r, i} \mathbf{B}_{i} \mathbf{s}_{i}+\mathbf{v}_{r}
$$

where $\mathbf{H}_{r, i}, i=1,2$, is the $M \times N$ channel matrix from source $i$ to the relay node and $\mathbf{v}_{r}$ is the $M \times 1$ noise vector at the relay node.

At the second time slot, $\mathbf{y}_{r}$ is linearly precoded at the relay node as

$$
\mathbf{x}_{r}=\mathbf{F} \mathbf{y}_{r}
$$

where $\mathbf{F}$ is the $M \times M$ relay precoding matrix. The relay node then broadcasts $\mathbf{x}_{r}$ to two destination nodes. The received signal vector at destination $i$ can be written as

$$
\begin{aligned}
\mathbf{y}_{i} & =\mathbf{H}_{i, r} \mathbf{x}_{r}+\mathbf{v}_{i} \\
& =\mathbf{H}_{i, r} \mathbf{F} \sum_{i=1}^{2} \mathbf{H}_{r, i} \mathbf{B}_{i} \mathbf{s}_{i}+\mathbf{H}_{i, r} \mathbf{F} \mathbf{v}_{r}+\mathbf{v}_{i}, \quad i=1,2
\end{aligned}
$$


where $\mathbf{H}_{i, r}$ is the $N \times M$ channel matrix between the relay node and destination $i$, and $\mathbf{v}_{i}$ is the $N \times 1$ noise vector at destination $i$.

We assume that all channel matrices are quasi-static, i.e., they remain constant over one time block, but can change to another value in the next block. Such quasi-static channel model has been widely used in two-way MIMO relay communications [6]-[8]. Two destination nodes can know the channel state information (CSI) of $\mathbf{H}_{i, r}$ and $\mathbf{H}_{r, i}, i=1,2$, through channel training and estimation [12]. One of the source nodes takes the responsibility for the optimization of $\mathbf{B}_{1}, \mathbf{B}_{2}$, and $\mathbf{F}$, and then transmits them to other nodes. In this way, all nodes in the system have the information of $\mathbf{B}_{1}, \mathbf{B}_{2}$, and $\mathbf{F}$. We also assume that $E\left[\mathbf{s}_{i} \mathbf{s}_{i}^{H}\right]=\mathbf{I}_{N_{b}}, i=1,2$, where $E[\cdot]$ denotes the statistical expectation, $(\cdot)^{H}$ stands for the Hermitian transpose, and $\mathbf{I}_{n}$ denotes the $n \times n$ identity matrix. All noises are independent and identically distributed (i.i.d.) additive white Gaussian noise with zero mean and unit variance.

According to the assumptions and analysis above, the selfinterference (SI) term $\mathbf{H}_{i, r} \mathbf{F} \mathbf{H}_{r, i} \mathbf{B}_{i} \mathbf{s}_{i}$ in (3) can be easily removed, since all information on this term is known to destination $i$. The received signal vector after the SI removal can be written as

$$
\tilde{\mathbf{y}}_{i}=\overline{\mathbf{H}}_{i} \mathbf{s}_{i^{\prime}}+\overline{\mathbf{v}}_{i}, \quad i=1,2
$$

where $\overline{\mathbf{H}}_{i} \triangleq \mathbf{H}_{i, r} \mathbf{F H}_{r, i^{\prime}} \mathbf{B}_{i^{\prime}}$ is the equivalent MIMO channel between source $i^{\prime}$ and destination $i$, and $\overline{\mathbf{v}}_{i} \triangleq \mathbf{H}_{i, r} \mathbf{F} \mathbf{v}_{r}+\mathbf{v}_{i}$ is the equivalent noise vector at destination $i$. Here $i^{\prime}=1$ for $i=2$, and $i^{\prime}=2$ for $i=1$.

Due to their low computational complexity, linear receivers are used at the destination nodes to retrieve the transmitted signals. Denoting $\mathbf{W}_{i}$ as the $N \times N_{b}$ weight matrix at destination $i$, the estimated signal vector is given by

$$
\hat{\mathbf{s}}_{i^{\prime}}=\mathbf{W}_{i}^{H} \tilde{\mathbf{y}}_{i}, \quad i=1,2 .
$$

\section{Proposed Algorithm With QoS Constraints}

In this section, we develop an iterative algorithm to optimize the source precoding matrices $\mathbf{B}_{1}, \mathbf{B}_{2}$, the relay precoding matrix $\mathbf{F}$, and the destination receive matrices $\mathbf{W}_{1}, \mathbf{W}_{2}$ to minimize the total transmission power of the source and relay nodes when certain QoS constraints are satisfied. From (1) and (2), the transmission power consumed by the relay node is given by

$$
P_{r}=\operatorname{tr}\left(\mathbf{F}\left(\mathbf{H}_{r, 1} \mathbf{B}_{1} \mathbf{B}_{1}^{H} \mathbf{H}_{r, 1}^{H}+\mathbf{H}_{r, 2} \mathbf{B}_{2} \mathbf{B}_{2}^{H} \mathbf{H}_{r, 2}^{H}+\mathbf{I}_{M}\right) \mathbf{F}^{H}\right)
$$

where $\operatorname{tr}(\cdot)$ denotes the matrix trace. The transmission power consumed by the source node $i$ is

$$
P_{i}=\operatorname{tr}\left(\mathbf{B}_{i} \mathbf{B}_{i}^{H}\right), \quad i=1,2 .
$$

The MSE of the signal waveform estimation at destination $i$ is given by

$$
\mathrm{MSE}_{i}=\operatorname{tr}\left(E\left[\left(\hat{\mathbf{s}}_{i^{\prime}}-\mathbf{s}_{i^{\prime}}\right)\left(\hat{\mathbf{s}}_{i^{\prime}}-\mathbf{s}_{i^{\prime}}\right)^{H}\right]\right), \quad i=1,2 .
$$

By substituting (4) and (5) into (8), we obtain that

$$
\mathrm{MSE}_{i}=\operatorname{tr}\left(\left(\mathbf{W}_{i}^{H} \overline{\mathbf{H}}_{i}-\mathbf{I}_{N_{b}}\right)\left(\mathbf{W}_{i}^{H} \overline{\mathbf{H}}_{i}-\mathbf{I}_{N_{b}}\right)^{H}+\mathbf{W}_{i}^{H} \mathbf{C}_{i} \mathbf{W}_{i}\right)
$$

where $\mathbf{C}_{i} \triangleq E\left[\overline{\mathbf{v}}_{i} \overline{\mathbf{v}}_{i}^{H}\right]=\mathbf{H}_{i, r} \mathbf{F} \mathbf{F}^{H} \mathbf{H}_{i, r}^{H}+\mathbf{I}_{N}$ is the covariance matrix of $\overline{\mathbf{v}}_{i}$.

From (6)-(9), the joint source, relay, and receive matrices optimization problem for two-way MIMO relay system with QoS constraints can be written as

$$
\begin{aligned}
& \min _{\mathbf{W}_{1}, \mathbf{W}_{2}, \mathbf{B}_{1}, \mathbf{B}_{2}, \mathbf{F}} P_{r}+P_{1}+P_{2} \\
& \text { s.t. } \mathrm{MSE}_{i} \leq e_{i}, \quad i=1,2
\end{aligned}
$$

where (10) is the objective function of the total transmission power in the system, and $e_{i}>0$ defines the upper-bound of $\mathrm{MSE}_{i}$. In practical systems, MSE effectively measures the accuracy of the signal waveform estimation at the receivers. Moreover, MSE is directly related to other commonly used QoS criteria such as the system BER and the sourcedestination mutual information [9]. The problem (10)-(11) is nonconvex with matrix variables, and the globally optimal solution is computationally intractable to obtain. Hereafter, we develop a computationally efficient algorithm to optimize the source, relay, and receive matrices in an iterative fashion through solving convex subproblems.

\section{A. Optimal Receive Matrices}

Since $\mathbf{W}_{1}$ and $\mathbf{W}_{2}$ do not appear in the objective function (10), for any fixed $\mathbf{B}_{1}, \mathbf{B}_{2}$, and $\mathbf{F}$, we choose $\mathbf{W}_{i}$ to minimize $\mathrm{MSE}_{i}$ in (9). The optimal $\mathbf{W}_{i}$ is the Wiener filter given by

$$
\mathbf{W}_{i}=\left(\overline{\mathbf{H}}_{i} \overline{\mathbf{H}}_{i}^{H}+\mathbf{C}_{i}\right)^{-1} \overline{\mathbf{H}}_{i}, \quad i=1,2
$$

where $(\cdot)^{-1}$ denotes the matrix inversion.

\section{B. Relay Matrix Optimization}

With fixed $\left(\mathbf{W}_{1}, \mathbf{W}_{2}\right)$ and $\left(\mathbf{B}_{1}, \mathbf{B}_{2}\right)$, we reformulate the problem (10)-(11) as a semi-definite programming (SDP) problem to optimize $\mathbf{F}$. By introducing

$$
\mathbf{H}_{i} \triangleq \mathbf{H}_{r, i^{\prime}} \mathbf{B}_{i^{\prime}}, \quad \mathbf{G}_{i} \triangleq \mathbf{W}_{i}^{H} \mathbf{H}_{i, r}, \quad i=1,2
$$

and using the following identities

$$
\begin{aligned}
& \operatorname{tr}\left(\mathbf{A}^{T} \mathbf{B}\right)=(\operatorname{vec}(\mathbf{A}))^{T} \operatorname{vec}(\mathbf{B}) \\
& \operatorname{tr}\left(\mathbf{A}^{H} \mathbf{B} \mathbf{A C}\right)=\operatorname{vec}(\mathbf{A})^{H}\left(\mathbf{C}^{T} \otimes \mathbf{B}\right) \operatorname{vec}(\mathbf{A})
\end{aligned}
$$

where $(\cdot)^{T}$ denotes the matrix transpose, $\otimes$ denotes the matrix Kronecker product and $\operatorname{vec}(\mathbf{X})$ stands for a column vector obtained by stacking all columns of $\mathbf{X}$ on top of each other, we obtain that

$$
\begin{aligned}
& \operatorname{tr}\left(\mathbf{W}_{i}^{H} \overline{\mathbf{H}}_{i} \overline{\mathbf{H}}_{i}^{H} \mathbf{W}_{i}\right)=\operatorname{tr}\left(\mathbf{G}_{i} \mathbf{F} \mathbf{H}_{i} \mathbf{H}_{i}^{H} \mathbf{F}^{H} \mathbf{G}_{i}^{H}\right) \\
& =\mathbf{f}^{H}\left(\left(\mathbf{H}_{i} \mathbf{H}_{i}^{H}\right)^{T} \otimes\left(\mathbf{G}_{i}^{H} \mathbf{G}_{i}\right)\right) \mathbf{f}
\end{aligned}
$$

where $\mathbf{f} \triangleq \operatorname{vec}(\mathbf{F})$. By substituting (15)-(17) back into (9), $\mathrm{MSE}_{i}$ can be expressed as

$$
\mathrm{MSE}_{i}=\mathbf{f}^{H} \mathbf{A}_{i} \mathbf{f}-\mathbf{c}_{i}^{H} \mathbf{f}-\mathbf{f}^{H} \mathbf{c}_{i}+t_{i}
$$

where $\mathbf{A}_{i} \triangleq\left(\left(\mathbf{H}_{i} \mathbf{H}_{i}^{H}\right)^{T}+\mathbf{I}_{M}\right) \otimes\left(\mathbf{G}_{i}^{H} \mathbf{G}_{i}\right), \mathbf{c}_{i} \triangleq \operatorname{vec}\left(\mathbf{G}_{i}^{H} \mathbf{H}_{i}^{H}\right)$, and $t_{i} \triangleq \operatorname{tr}\left(\mathbf{W}_{i}^{H} \mathbf{W}_{i}\right)+N_{b}$. 
Substituting (13) back into (6) and using (14), we have

$$
P_{r}=\mathbf{f}^{H}\left(\left(\mathbf{H}_{2} \mathbf{H}_{2}^{H}+\mathbf{H}_{1} \mathbf{H}_{1}^{H}+\mathbf{I}_{M}\right)^{T} \otimes \mathbf{I}_{M}\right) \mathbf{f} .
$$

Note that we can ignore the term $P_{1}+P_{2}$ in (10) when optimizing $\mathbf{F}$ with given $\left(\mathbf{W}_{1}, \mathbf{W}_{2}\right)$ and $\left(\mathbf{B}_{1}, \mathbf{B}_{2}\right)$, since it is free of the optimization variable $\mathbf{F}$. By introducing a new auxiliary variable $p$, which satisfies $p \geq \mathbf{f}^{H} \mathbf{\Phi f}$ with $\boldsymbol{\Phi} \triangleq\left(\mathbf{H}_{2} \mathbf{H}_{2}^{H}+\mathbf{H}_{1} \mathbf{H}_{1}^{H}+\mathbf{I}_{M}\right)^{T} \otimes \mathbf{I}_{M}$, and using the Schur complement, the problem (10)-(11) can be equivalently rewritten as the following SDP problem

$$
\begin{aligned}
\min _{p, \mathbf{f}} p & \\
\text { s.t. } & {\left[\begin{array}{cc}
p & \mathbf{f}^{H} \\
\mathbf{f} & \mathbf{\Phi}^{-1}
\end{array}\right] \succeq 0 } \\
& {\left[\begin{array}{cc}
e_{i}-t_{i}+\mathbf{c}_{i}^{H} \mathbf{f}+\mathbf{f}^{H} \mathbf{c}_{i} & \mathbf{f}^{H} \mathbf{A}_{i}^{\frac{1}{2}} \\
\left(\mathbf{A}_{i}^{\frac{1}{2}}\right)^{H} \mathbf{f} & \mathbf{I}_{M^{2}}
\end{array}\right] \succeq 0, i=1,2(21) }
\end{aligned}
$$

where for a matrix $\mathbf{A}, \mathbf{A} \succeq 0$ means that $\mathbf{A}$ is a positive semidefinite (PSD) matrix. The problem (19)-(21) can be efficiently solved by the disciplined convex programming toolbox CVX [13].

\section{Source Matrices Optimization}

With given $\mathbf{W}_{i}$ and $\mathbf{F}, \mathbf{B}_{i}$ can be optimized by solving the following problem

$$
\begin{aligned}
& \min _{\mathbf{B}_{i}} \operatorname{tr}\left(\mathbf{B}_{i}^{H}\left(\mathbf{I}_{N}+\mathbf{H}_{r, i}^{H} \mathbf{F}^{H} \mathbf{F} \mathbf{H}_{r, i}\right) \mathbf{B}_{i}\right) \\
& \text { s.t. } \operatorname{tr}\left(\left(\tilde{\mathbf{H}}_{i} \mathbf{B}_{i}-\mathbf{I}_{N_{b}}\right)\left(\tilde{\mathbf{H}}_{i} \mathbf{B}_{i}-\mathbf{I}_{N_{b}}\right)^{H}\right) \leq \tilde{e}_{i^{\prime}}
\end{aligned}
$$

where $\tilde{e}_{i^{\prime}} \triangleq e_{i^{\prime}}-\operatorname{tr}\left(\mathbf{W}_{i^{\prime}}^{H}\left(\mathbf{H}_{i^{\prime}, r} \mathbf{F} \mathbf{F}^{H} \mathbf{H}_{i^{\prime}, r}^{H}+\mathbf{I}_{N}\right) \mathbf{W}_{i^{\prime}}\right), \tilde{\mathbf{H}}_{i} \triangleq$ $\mathbf{W}_{i^{\prime}}^{H} \mathbf{H}_{i^{\prime}, r} \mathbf{F H}_{r, i}$. By introducing $\mathbf{D}_{i} \triangleq \mathbf{I}_{N}+\mathbf{H}_{r, i}^{H} \mathbf{F}^{H} \mathbf{F} \mathbf{H}_{r, i}$ and applying the Lagrange multiplier method to the problem (22)-(23), we obtain

$$
\begin{aligned}
\mathcal{L}_{i}= & \operatorname{tr}\left(\mathbf{B}_{i}^{H} \mathbf{D}_{i} \mathbf{B}_{i}+\mu\left(\mathbf{B}_{i}^{H} \tilde{\mathbf{H}}_{i}^{H} \tilde{\mathbf{H}}_{i} \mathbf{B}_{i}\right.\right. \\
& \left.\left.-\tilde{\mathbf{H}}_{i} \mathbf{B}_{i}-\mathbf{B}_{i}^{H} \tilde{\mathbf{H}}_{i}^{H}+\mathbf{I}_{N_{b}}\right)\right)-\mu \tilde{e}_{i^{\prime}}
\end{aligned}
$$

where $\mu \geq 0$ is the Lagrangian multiplier. By taking the derivative of (24) with respect to $\mathbf{B}_{i}$, we obtain

$$
\mathbf{B}_{i}=\mu\left(\mathbf{D}_{i}+\mu \tilde{\mathbf{H}}_{i}^{H} \tilde{\mathbf{H}}_{i}\right)^{-1} \tilde{\mathbf{H}}_{i}^{H} .
$$

The Lagrangian multiplier $\mu$ in (25) can be found from the following complementary slackness condition

$$
\mu\left(\operatorname{tr}\left(\left(\tilde{\mathbf{H}}_{i} \mathbf{B}_{i}-\mathbf{I}_{N_{b}}\right)\left(\tilde{\mathbf{H}}_{i} \mathbf{B}_{i}-\mathbf{I}_{N_{b}}\right)^{H}\right)-\tilde{e}_{i^{\prime}}\right)=0 .
$$

Obviously, (26) holds if $\mu=0$. However, this results in $\mathbf{B}_{i}=$ $\mathbf{0}$. Thus, there must be $\mu>0$, and $\mathbf{B}_{i}$ in (25) must satisfy

$$
\operatorname{tr}\left(\left(\tilde{\mathbf{H}}_{i} \mathbf{B}_{i}-\mathbf{I}_{N_{b}}\right)\left(\tilde{\mathbf{H}}_{i} \mathbf{B}_{i}-\mathbf{I}_{N_{b}}\right)^{H}\right)=\tilde{e}_{i^{\prime}} .
$$

Let us introduce the singular value decomposition (SVD) of $\tilde{\mathbf{H}}_{i}=\mathbf{U}_{i} \boldsymbol{\Lambda}_{i} \mathbf{V}_{i}^{H}$, where the dimensions of $\mathbf{U}_{i}, \boldsymbol{\Lambda}_{i}$, and $\mathbf{V}_{i}$ are $N_{b} \times N_{b}, N_{b} \times N_{b}$, and $N \times N_{b}$, respectively. By substituting (25) into (27), we obtain

$$
\operatorname{tr}\left(\left(\boldsymbol{\Lambda}_{i}\left(\tilde{\mathbf{D}}_{i} / \mu+\boldsymbol{\Lambda}_{i}^{2}\right)^{-1} \boldsymbol{\Lambda}_{i}-\mathbf{I}_{N_{b}}\right)^{2}\right)=\tilde{e}_{i^{\prime}}
$$

TABLE I

Procedure of The Proposed Algorithm

1) Initialize the algorithm at feasible $\mathbf{B}_{1}^{(0)}, \mathbf{B}_{2}^{(0)}$, and $\mathbf{F}^{(0)}$; set $n=0$.

2) Update $\mathbf{W}_{1}^{(n)}$ and $\mathbf{W}_{2}^{(n)}$ with fixed $\mathbf{B}_{1}^{(n)}, \mathbf{B}_{2}^{(n)}$, and $\mathbf{F}^{(n)}$, as shown in (12).

3) Solve the SDP problem (19)-(21) using given $\mathbf{W}_{1}^{(n)}, \mathbf{W}_{2}^{(n)}$ and $\mathbf{B}_{1}^{(n)}, \mathbf{B}_{2}^{(n)}$ to obtain $\mathbf{F}^{(n+1)}$.

4) Obtain $\mathbf{B}_{1}^{(n+1)}, \mathbf{B}_{2}^{(n+1)}$ with known $\mathbf{W}_{1}^{(n)}, \mathbf{W}_{2}^{(n)}$ and $\mathbf{F}^{(n+1)}$ according to (25).

5) If $\max \left|\mathbf{F}^{(n+1)}-\mathbf{F}^{(n)}\right|<\varepsilon$, then end; otherwise set $n=n+1$ and go to step 2).

where $\tilde{\mathbf{D}}_{i} \triangleq \mathbf{V}_{i}^{H} \mathbf{D}_{i} \mathbf{V}_{i}$. By introducing the eigenvalue decomposition of $\boldsymbol{\Lambda}_{i}^{-1} \tilde{\mathbf{D}}_{i} \boldsymbol{\Lambda}_{i}^{-1}=\mathbf{Q}_{i} \boldsymbol{\Sigma}_{i} \mathbf{Q}_{i}^{H}$, the left-hand side of (28) can be written as

$$
g_{i}(\mu) \triangleq \operatorname{tr}\left(\left(\left(\boldsymbol{\Sigma}_{i} / \mu+\mathbf{I}_{N_{b}}\right)^{-1}-\mathbf{I}_{N_{b}}\right)^{2}\right)=\sum_{k=1}^{N_{b}} \frac{\sigma_{i, k}^{2}}{\left(\sigma_{i, k}+\mu\right)^{2}}
$$

where $\sigma_{i, k}, k=1, \cdots, N_{b}$, is the $k$ th diagonal element of $\boldsymbol{\Sigma}_{i}$. It can be clearly seen that $g_{i}(\mu)$ is monotonically decreasing with respect to $\mu$ when $\mu>0$. Thus, the unique solution of (28) can be obtained by the bisection method.

Now the original joint source, relay, and receive matrices optimization problem (10)-(11) can be solved using the proposed iterative algorithm. This algorithm starts at random feasible $\mathbf{B}_{1}, \mathbf{B}_{2}$, and $\mathbf{F}$. At each iteration, we first optimize $\mathbf{W}_{1}$ and $\mathbf{W}_{2}$ based on $\mathbf{B}_{1}, \mathbf{B}_{2}$, and $\mathbf{F}$ from the previous iteration using (12). Then we update $\mathbf{F}$ with given $\mathbf{B}_{i}$ and $\mathbf{W}_{i}, i=1,2$, by solving the problem (19)-(21). Finally, we optimize $\mathbf{B}_{1}$ and $\mathbf{B}_{2}$ based on known $\mathbf{W}_{1}, \mathbf{W}_{2}$, and $\mathbf{F}$ according to (25). We continuously update five matrices in an alternating fashion till convergence. The procedure of the proposed algorithm is summarized in Table I, where the superscript $(n)$ denotes the number of iterations, $\max |\cdot|$ denotes the maximum among the absolute value of all elements in a matrix, and $\varepsilon$ is a positive constant close to 0 . Note that each step of optimization may either decrease or maintain but cannot increase the objective function (10). Moreover, the objective function is lower bounded by at least zero. Thus, the proposed algorithm converges.

At the convergence point, since $\mathbf{F}^{(n)}$ is the optimal solution to the relay matrix optimization problem (19)-(21), and $\mathbf{B}_{i}^{(n)}$ is the optimal solution to the source matrix optimization problem (22)-(23), we obtain that

$$
\begin{aligned}
& \operatorname{tr}\left(\nabla_{\mathbf{F}} J\left(\boldsymbol{\Theta}^{(n)}\right)^{T}\left(\mathbf{F}-\mathbf{F}^{(n)}\right)\right) \geq 0 \\
& \operatorname{tr}\left(\nabla_{\mathbf{B}_{i}} J\left(\mathbf{\Theta}^{(n)}\right)^{T}\left(\mathbf{B}_{i}-\mathbf{B}_{i}^{(n)}\right)\right) \geq 0, \quad i=1,2
\end{aligned}
$$

where $\boldsymbol{\Theta}^{(n)} \triangleq\left[\mathbf{F}^{(n)}, \mathbf{B}_{1}^{(n)}, \mathbf{B}_{2}^{(n)}\right]$ and $\nabla_{\mathbf{A}} J\left(\boldsymbol{\Theta}^{(n)}\right)$ is the gradient of the objective function (10) along the direction of $\mathbf{A} \in\left\{\mathbf{B}_{1}, \mathbf{B}_{2}, \mathbf{F}\right\}$ at $\boldsymbol{\Theta}^{(n)}$. Summing up (29) and (30), we have $\operatorname{tr}\left(\nabla J\left(\boldsymbol{\Theta}^{(n)}\right)^{T}\left(\boldsymbol{\Theta}-\boldsymbol{\Theta}^{(n)}\right)\right) \geq 0$, where $\nabla J\left(\boldsymbol{\Theta}^{(n)}\right) \triangleq\left[\nabla_{\mathbf{F}} J\left(\boldsymbol{\Theta}^{(n)}\right), \nabla_{\mathbf{B}_{1}} J\left(\mathbf{\Theta}^{(n)}\right), \nabla_{\mathbf{B}_{2}} J\left(\boldsymbol{\Theta}^{(n)}\right)\right]$, indicating that $\Theta^{(n)}$ is (at least) a stationary point of (10). As the optimization problem (10)-(11) is nonconvex with matrix variables, the globally optimal solution is intractable. Therefore, we cannot compute the gap between the solution obtained by the proposed algorithm and the globally optimal solution. 


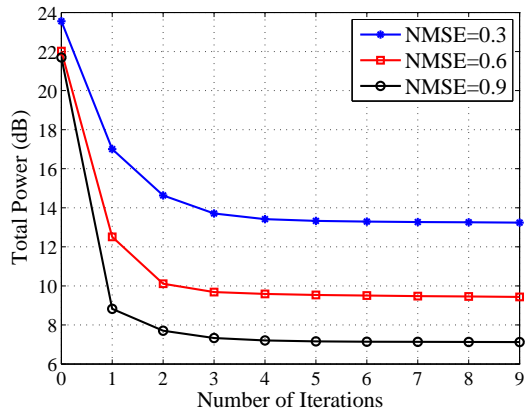

Fig. 2. Total power $(\mathrm{dB})$ versus number of iterations.

In each iteration of the proposed algorithm, the major computation task lies in solving the SDP problem (19)(21) to update $\mathbf{F}$, which has a worst-case complexity order of $\mathcal{O}\left(\left(M^{2}+1\right)^{4.5}\right)$ [14]. Since the amount of computation required for updating $\mathbf{W}_{i}$ and $\mathbf{B}_{i}, i=1,2$, is negligible compared with that of solving the SDP problem, the per-iteration complexity of the proposed algorithm is $\mathcal{O}\left(\left(M^{2}+1\right)^{4.5}\right)$. With the advancement of modern chip design, this amount of computation can be handled by the source nodes.

\section{NumericAl EXAMPLES}

In this section, we study the performance of the proposed algorithm through numerical simulations. All channel matrices have complex Gaussian entries with zero mean and unit variance, while $\mathbf{F}$ and $\mathbf{B}_{i}$ are initialized as random matrices with complex Gaussian entries of zero mean and variance of 0.5 and 2 , respectively. We assume that $\mathrm{MSE}_{1}=\mathrm{MSE}_{2}$ and normalize the MSE (9) by the number of data streams to obtain the normalized MSE (NMSE). All simulation results are averaged over 1000 independent channel realizations.

In the first example, we investigate the convergence speed of the proposed algorithm. We simulate a two-way MIMO relay system with $N_{b}=N=2$, and $M=4$. Fig. 2 shows the total transmission power versus the number of iterations when the NMSE is set to $0.3,0.6$, and 0.9 , respectively. It can be seen from Fig. 2 that the proposed algorithm converges typically within ten iterations. In fact, the decreasing of the total power after four iterations is very small. Therefore, only a small number of iterations are required to achieve a good performance. This indicates that the proposed algorithm has a low computational complexity and short delay, which is important for practical two-way MIMO relay systems. It can also be seen from Fig. 2 that when the MSE constraints become stricter, more transmission power is needed to meet stricter QoS requirements, which reflects the typical QoS-cost tradeoff in communication systems.

In the second example, we compare the total power versus the NMSE of the proposed iterative algorithm, the naive amplify-and-forward (NAF) algorithm where $\mathbf{B}_{1}, \mathbf{B}_{2}$, and $\mathbf{F}$ are scaled identity matrices satisfying the MSE constraints, and the SVD-based algorithm in [8]. We set $N_{b}=N=2$. It can be clearly seen from Fig. 3 that the proposed algorithm requires much less total power than the NAF algorithm and the SVD-based algorithm. We also observe that using the proposed

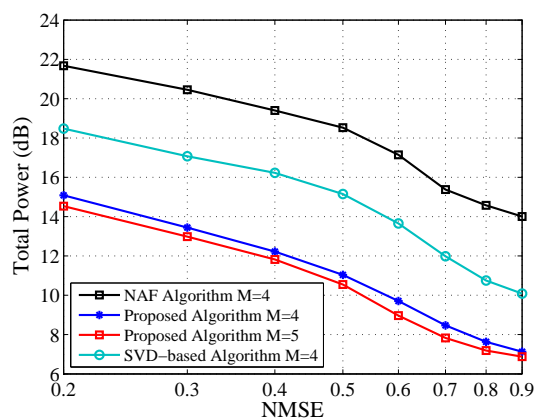

Fig. 3. Total power (dB) versus NMSE with different $M$.

algorithm, the system with $M=5$ needs less total power than the system with $M=4$.

\section{CONCLUSIONS}

We investigated the QoS issue in two-way MIMO relay systems. An iterative algorithm has been developed to optimize the source, relay, and receive matrices to minimize the total transmission power when QoS-constraints on MSE are satisfied. The fast convergence of the proposed algorithm has been demonstrated through numerical simulations.

\section{REFERENCES}

[1] A. Nosratinia, T. E. Hunter, and A. Hedayat, "Cooperative communication in wireless networks," IEEE Commun. Magazine, vol. 42, pp. 74-80, Oct. 2004.

[2] B. Wang, J. Zhang, and A. Høst-Madsen, "On the capacity of MIMO relay channels," IEEE Trans. Inf. Theory, vol. 51, pp. 29-43, Jan. 2005.

[3] L. Sanguinetti, A. A. D'Amico, and Y. Rong, "A tutorial on the optimization of amplify-and-forward MIMO relay systems," IEEE J. Select. Areas Commun., vol. 30, pp. 1331-1346, Sep. 2012.

[4] Z. Chen, T. J. Lim, and M. Motani. "Digital network coding aided twoway relaying: Energy minimization and queue analysis," IEEE Trans. Wireless Commun., vol. 12, pp. 1947-1957, Apr. 2013.

[5] M. P. Wilson and K. R. Narayanan, "Power allocation strategies and lattice based coding schemes for bidirectional relaying," in Proc. ISIT, Seoul, Korea, June 28-July 3, 2009, pp. 344-348.

[6] S. Xu and Y. Hua, "Optimal design of spatial source-and-relay matrices for a non-regenerative two-way MIMO relay system," IEEE Trans. Wireless Commun., vol. 10, pp. 1645-1655, May 2011.

[7] R. Wang, M. Tao, and Y. Huang, "Linear precoding designs for amplifyand-forward multiuser two-way relay systems," IEEE Trans. Wireless Commun., vol. 11, pp. 4457-4469, Dec. 2012.

[8] Y. Rong, "Joint source and relay optimization for two-way linear non-regenerative MIMO relay communications," IEEE Trans. Signal Process., vol. 60, pp. 6533-6546, Dec. 2012.

[9] L. Sanguinetti, A. A. D'Amico, and Y. Rong, "On the design of amplifyand-forward MIMO-OFDM relay systems with QoS requirements specified as Schur-convex functions of the MSEs," IEEE Trans. Veh. Technol., vol. 62, pp. 1871-1877, May 2013.

[10] Y. Fu, L. Yang, W.-P. Zhu, and C. Liu, "Optimum linear design of twohop MIMO relay networks with QoS requirements," IEEE Trans. Signal Process., vol. 59, pp. 2257-2269, May 2011.

[11] L. Sanguinetti and A. A. D'Amico, "Power allocation in two-hop amplify-and-forward MIMO relay systems with QoS requirements," IEEE Trans. Signal Process., vol. 60, pp. 2494-2507, May 2012.

[12] C. W. R. Chiong, Y. Rong, and Y. Xiang, "Channel training algorithms for two-way MIMO relay systems,” IEEE Trans. Signal Process., vol. 61 pp. 3988-3998, Aug. 2013.

[13] M. Grant and S. Boyd, "CVX: Matlab software for disciplined convex programming (web page and software)," Apr. 2010. Available: http://cvxr.com/cvx.

[14] M. Lobo, L. Vandenberghe, S. Boyd, and H. Lebret, "Application of second-order cone programming," Linear Algebra and Its Applications, vol. 284, no. 13, pp. 193-228, Nov. 1998. 